\title{
Entrelacs
}

ENTRELACS Cinéma et audiovisuel

$10 \mid 2013$

Le Toucher

\section{Quelle poétique de la main dans les jeux vidéo?}

\section{Thomas Morisset}

\section{(2) OpenEdition \\ Journals}

Édition électronique

URL : http://journals.openedition.org/entrelacs/495

DOI : 10.4000/entrelacs.495

ISSN : 2261-5482

Éditeur

Éditions Téraèdre

\section{Référence électronique}

Thomas Morisset, «Quelle poétique de la main dans les jeux vidéo ? », Entrelacs [En ligne], 10 | 2013, mis en ligne le 11 septembre 2013, consulté le 01 mai 2019. URL : http://journals.openedition.org/ entrelacs/495; DOI : 10.4000/entrelacs.495

Ce document a été généré automatiquement le 1 mai 2019.

Tous droits réservés 


\title{
Quelle poétique de la main dans les jeux vidéo?
}

\author{
Thomas Morisset
}

1 S'intéresser à la place de la main dans les jeux vidéo revient à considérer deux objets différents, quoique complémentaires : la main du joueur qui appuie sur la manette, et la main du personnage virtuel qui accomplit tout ce que le programme informatique veut bien lui laisser accomplir ; deux objets qui requièrent des approches problématiques différentes.

Dans le cas de la main virtuelle, il faudra tout simplement se demander en quoi celle-ci, certes modélisée de manière de plus en plus réaliste, est véritablement une main ou bien un simple habillage. Est-elle capable de simuler le toucher, d'avoir dans son environnement virtuel le même rôle qu'elle a pour nous dans le monde physique ? Nous ne parlerons pas ici des scènes cinématiques qui empruntent une grande partie de leur mise en scène au septième art, mais uniquement des sections de jeu dans lesquels le joueur a une action tangible. Par action tangible, nous n'entendons pas autre chose que l'usage par le joueur de sa main pour appuyer sur les boutons de la manette. Ces gestes, ne sont-ils que purement instrumentaux ou bien ont-ils une valeur esthétique ? Plus précisément, cela pose la question du degré de participation du toucher et du corps à l'expérience vidéoludique : l'interaction n'est-elle qu'un gadget qui ferait du jeu vidéo un cinéma interactif ou bien joue-t-elle un rôle plus profond dans la constitution de l'expérience?

\section{La main de l'avatar : outil et cosmétique}

3 Distinguons d'abord deux types de jeux, ceux dans lesquels le joueur accomplit des actions vidéoludiques en restant une instance abstraite ou absente dans l'univers virtuel (comme dans Tétris par exemple) et ceux dans lesquels ce pouvoir d'agir est rattaché à un avatar, terme qui désigne le personnage fictionnel que le joueur est amené à diriger dans l'univers du jeu (ainsi la série des Super Mario). Dans cette dernière catégorie, le lien visuel 
entre le joueur et son avatar peut s'établir selon deux modalités : via une caméra à la troisième personne, lorsque le point de vue est situé dans le dos du personnage, ou bien via une caméra à la première personne, lorsque l'on voit à travers les yeux de l'avatar.

Que veut alors dire "diriger un avatar »? On emploie souvent le terme "incarner", comme pour signifier que l'on prend possession et contrôle du corps de celui-ci. Le problème est que ce contact avec le corps, s'il a lieu visuellement dans le jeu à la troisième personne, n'a lieu que de manière incomplète dans le cas d'un jeu en caméra subjective. Le corps de l'avatar est deviné, soupçonné, suppléé par l'imagination et l'habitude du joueur. Seul les miroirs et les cinématiques nous rappellent son apparence. Remarquons déjà toute la difficulté à envisager une expérience tactile si le référent dans le monde virtuel est un corps qu'on ne sent, ni ne voit, bref avec lequel le contact n'est que purement du domaine de l'action.

5 Prenons pour exemple le jeu Mirror's Edge $e^{1}$, dans lequel on dirige, à la première personne, Faith, jeune femme devant sauter de toits en toits dans une cité futuriste aux relents totalitaires. Lorsque celle-ci grimpe ou fait une roulade, ses mains apparaissent à l'écran pour attraper telle ou telle corniche ou bien pour amortir sa chute. La première de ces actions se déclenche automatiquement lorsque Faith approche d'un rebord ou d'une échelle, quand la seconde demande au joueur d'appuyer sur une touche au moment opportun. Mais, dans les deux cas, nous ne pouvons pas dire que le joueur contrôle les mains de Faith. On peut comprendre cela, négativement, si l'on étudie la même situation dans un autre jeu. Ainsi, dans Half-Life $2^{2}$, Gordon Freeman, l'avatar, peut lui aussi monter sur des échelles, mais sans que ses mains n'apparaissent à l'écran. L'action accomplie par Freeman n'en est pas pour autant incompréhensible : le joueur sait qu'il dirige un humain et qu'un humain peut monter aux échelles. On peut à la rigueur déplorer l'absence d'animation des bras et des mains, qui donne au fond l'impression que le personnage flotte devant l'échelle, mais c'est la même fonction qui, dans les deux jeux, est parfaitement accomplie. La main n'est donc ici que cosmétique, elle est un habillage donnant une plus grande beauté à l'expérience de jeu. L'avatar est dirigé comme un tout abstrait et compact, il est pure fonction et seul le regard est autonome par rapport à ce bloc de mouvements qui peuvent, ou non, être rendus à l'écran par des animations.

Qu'est-ce qu'il manque aux mains de Faith pour que leur toucher soit autre chose qu'un toucher accidentel et purement fonctionnel ? André Leroi-Gourhan note dans La mémoire et les rythmes que la spécificité de la main humaine est qu'elle n'est plus un outil mais ce qui fabrique, ce qui meut les outils ${ }^{3}$. En exportant ses gestes dans des outils, la main se libère et devient pleinement humaine, c'est-à-dire autonome, ne se spécialisant pas dans une tâche particulière. Or on voit que la main de l'avatar, parce qu'elle est liée à la fonction ludique de celui-ci, n'a pas cette autonomie et demeure l'outil extériorisé de la main autonome du joueur, assis derrière l'écran. Un toucher esthétique virtuel ne pourrait alors advenir que si la main gagne une part de cette autonomie au sein du monde fictionnel du jeu vidéo.

\section{L.A. Noire ou le toucher optique}

7 L'un des rares jeux dans lequel il nous est donné la possibilité de contrôler, de façon indépendante, la main d'un avatar est le récent L.A. Noire ${ }^{4}$. Dans celui-ci, le joueur incarne Cole Phelps, inspecteur de police dans le Los Angeles de l'après-guerre. Celui-ci devra résoudre toute une série d'enquêtes criminelles en ayant recours à des séances 
d'interrogatoires et à la collecte d'indices matériels sur les lieux du crime ou dans les appartements des suspects.

Lors de ces phases, Phelps peut ramasser toutes sortes d'objets et les manipuler afin de faire apparaître un élément pertinent pour l'enquête. Cependant, ces manipulations ne s'apparentent pas encore à du toucher, au sens où ce ne sont pas les propriétés tactiles de l'objet qui sont sources de plaisir ou d'intérêt esthétique. En effet, si l'avatar dispose d'une plus grande liberté de sa main virtuelle, parce que, pour un temps, le contrôle est véritablement dans la main, celle-ci est malgré tout entraînée dans un rapport optique à ce qu'elle touche. La manipulation est une mise en lumière pour l'œil, ce qui se manifeste par le fait que, lorsque le point pertinent de l'objet a été trouvé (la tache de sang au revers d'une statuette, un mot griffonné sur un paquet d'allumettes...), un zoom a lieu pendant que Phelps commente la découverte.

9 Cette subordination de la main à l'œil rejoint ici les analyses proposées par Gilles Deleuze et Félix Guattari dans Mille Plateaux lorsqu'ils distinguent l'espace optique de l'espace haptique ${ }^{5}$. Haptique/optique est le mode esthétique de compréhension de la dualité lisse/ strié, qui, parmi ses différentes expressions, nous semble le mieux résumée par cette phrase : «[...] alors que dans le strié les formes organisent une matière, dans le lisse des matériaux signalent des forces ou leur servent de symptômes. » ${ }^{6}$ Nous avons d'un côté une mise à distance par une instance, à la manière de l'œil qui ordonne le proche et le lointain en différents plans, de l'autre un contact qui ne marque pas de frontières, mais donne à ressentir des énergies, comme l'expérience tactile dans laquelle différentes textures, différentes surfaces se réunissent sur un même espace de contact aux contours assez flous.

Une telle subordination ne doit au fond pas surprendre ; le jeu vidéo est en soi un espace strié parce que lié au code. La strie propre qui mesure l'espace vidéoludique est celle des éléments jouables, interactifs et des éléments non-jouables qui ne sont que décors. Une des logiques qui préside à l'exploration de tels univers est la recherche et la mise en évidence de ce qui est pertinent, en dessous de l'enveloppe de pixels, que l'on peut résumer par la formule " pointe et clique " ${ }^{7}$. À l'inverse peut-être de notre monde qui est d'abord lisse avant d'être strié par l'homme, le monde virtuel est d'abord un champ de stries que l'image va tenter de lisser - la main de Faith n'est pas autre chose qu'un ornement destiné à rapprocher le joueur de son personnage.

11 Si Gilles Deleuze et Félix Guattari ont en effet situé l'espace haptique dans une relation de proximité, cette proximité est une qualité et n'est pas nécessairement synonyme de localité : selon eux, le désert est saisi par celui qui y transite sur le mode haptique, en ce qu'il efface les distances et constitue un lieu peu, voire pas, délimité ; l'immensité abolit les bordures et donne à sentir8. De même, l'apparence de la proximité physique peut en fait receler un éloignement : c'est précisément ce que montre L.A. Noire, les gros plans sur la main de Phelps ne sont pas gages de toucher. Le règne de l'optique est tel que c'est le toucher qui devient lui-même optique.

\section{La main humaine et la machine informatique}

12 Cette déperdition du toucher comme proximité a pourtant de quoi surprendre si l'on songe à l'histoire du jeu vidéo et de l'informatique en général. L'avènement de l'informatique s'est fait par la possibilité d'un rapport intime avec la machine, lorsqu'au 
début des années soixante les ordinateurs se sont dotés d'un écran et d'un clavier afin que l'on puisse coder en temps réel. ${ }^{9}$ Se forme alors une communauté d'étudiants adepte du hack, c'est-à-dire :

[...] une combinaison ingénieuse, une invention à laquelle personne n'avait encore songé, [...] un raccourci qui permet de faire plus vite et plus élégamment. C'est de la technique, mais élevé au rang d'art, appréciée pour sa valeur esthétique, son style, plutôt que pour son utilité. ${ }^{10}$

Un hack n'est donc possible que par un contact, par un toucher qui grise la frontière entre l'homme et la machine, bref par l'établissement d'un écosystème, d'un espace lisse qui unit les deux, par opposition à la carte striée qui devait être confectionnée à part avant d'être remise à l'utilisateur de l'ordinateur.

Une des réalisations du groupe de hacker du MIT fut alors, en 1962, Spacewars!, pionnier des jeux vidéo, dans lequel s'affrontent deux vaisseaux spatiaux autour d'une étoile exerçant sur eux sa force de gravité. ${ }^{11}$ Les jeux vidéo sont donc nés de cette effervescence du contact avec l'être nouveau qu'était à l'époque l'ordinateur et de l'appropriation créatrice de cette intimité technologique. Mais, maintenant que le joueur de jeux vidéo moyen n'entend rien ou presque rien à l'art du code, cet aspect créateur s'est effacé et ne survit, en somme, que dans la position du joueur, devant son écran, armé de sa manette.

Certes l'interaction humain/machine est toujours grande, mais celle-ci est réglée ; ce qui se traduit, du côté du joueur, par la nécessité d'incorporer un rythme : savoir sauter dans Mirror's Edge n'est pas simplement savoir appuyer sur un bouton, c'est savoir appuyer ni trop tôt, ni trop tard sur le bon bouton ou sur la bonne séquence de commandes. Qu'elles soient heurtées ou régulières, lentes ou effrénées, le jeu vidéo propose à la main du joueur des séquences rythmiques d'actions et de mouvements qui se traduisent le plus souvent à l'écran de manière spatiale.

Pour ces raisons, Graeme Kirkpatrick, dans son ouvrage Aesthetic Theory and the Videogame, fait de l'activité vidéoludique une nouvelle forme de danse ${ }^{12}$. Pour ce dernier, ce qui doit être premier dans l'étude esthétique du jeu vidéo, c'est le geste de la main parce qu'il est lui-même la part la plus primordiale de l'expérience vidéoludique. À propos de Mirror's Edge, l'auteur anglais note que :

[...] notre tâche est d'identifier les formes, ou les mouvements, que nous avons appris et de les utiliser pour progresser. En d'autres termes, le jeu est une chorégraphie (ou un script/une partition) et notre rôle de joueur est de suivre ses instructions. Nous ne pouvons faire cela de manière mécanique, mais devons trouver une réponse à l'aide notre imagination. ${ }^{13}$

18 Nous pensons néanmoins que le terme de « danse » ne convient pas aux mouvements des doigts du joueur. Graeme Kirkpatrick, en ramenant le jeu vidéo à de la danse méconnaît en fait le caractère spectaculaire de cette dernière. Or il n'y a pas de public pour regarder les mouvements d'une main sur une manette et ceux-ci ne sont d'ailleurs pas réalisés pour être regardés, mais pour produire un résultat qui, lui, soit digne d'être vu et apprécié ${ }^{14}$. Plutôt que de parler de danse, nous préférons alors parler de corps au travail.

\section{Le corps au travail et le sens du geste}

Pour comprendre ce que ce concept recouvre, pensons à un concert d'une petite formation, mettons un quatuor à cordes. Entre les notes, on percevra aisément les 
respirations des interprètes, voire, parfois, le bruit des doigts qui glissent le long des cordes, d'une position de main à une autre. Ces résidus, ces petits bruits participent du corps au travail rappellant que sous le monde symbolique façonné par la musique, il y a l'effort pour faire advenir et maintenir un tel monde - à charge, par la suite, à un interprète de les masquer ou non, à un compositeur de les intégrer dans une partition ou au public de les considérer ou non comme des parasites.

La pression de la main sur les boutons, le léger cliquetis que ceux-ci produisent, le corps... Ce sont les signes d'un corps au travail. Mais qu'est-ce qui est au juste créé par ce travail sous-jacent ? Non pas une œuvre d'art mais une expérience esthétique mêlant la poïétique et l'esthésique. Nous empruntons ces termes à Paul Valéry pour qui ils possèdent un sens catégoriel : ils constituent la science esthétique, l'esthésique se consacrant à «l'étude des sensations » quand la poḯtique s'intéresse aux conditions et moyens de la " production des œuvres $»^{15}$. Pour qualifier l'action du joueur, il ne suffit pas de dire qu'il façonne son expérience - regarder un film dans tel ou tel état d'esprit, lire en levant la tête sur tel ou tel mot, c'est déjà façonner l'expérience particulière d'une œuvre d'art. Mais changer la forme et le contenu de son expérience en agissant sur la forme ou sur le contenu du jeu vidéo, en actionnant, par exemple, telle commande plutôt que telle autre à un moment crucial, c'est s'engager dans une démarche poḯtique. En en restant là, le joueur de jeux vidéo ne serait pas différent d'un musicien qui interprète une œuvre, dotée de propriétés poïétiques propres à l'aide de sa dextérité et de " réactions sensibles » aux phrases musicales - créant un enchevêtrement entre les deux niveaux de l'esthétique selon Valéry. Mais si le musicien joue pour un public, le joueur de jeux vidéo est le seul récepteur possible d'une session de jeu. Cette différence de statut est également marquée par le fait qu'un jeu vidéo, contrairement à une œuvre musicale, répond, résiste en temps réel aux efforts du joueur et l'oblige en permanence à redéfinir son comportement, le poussant donc parfois à être dans une attitude de pure réception à l'œuvre pour pouvoir y tenir par la suite un rôle plus actif. Pour ces raisons, « esthésique " prend pour nous un sens plus précis : ce qui est ressenti par le ou les récepteurs d'une œuvre. Dans ce contexte, la main du joueur a donc quasi exclusivement un rôle poïétique, par opposition à une apparente prévalence esthésique de l'œil.

21 Au passage, il nous semble que le jeu à la manette est plus apte à créer des expériences nouvelles et satisfaisantes que la détection de mouvements : le sentiment de puissance est plus fort lorsqu'il passe dans la main car celle-ci devient la passerelle par laquelle notre corps entier acquiert un pouvoir physiquement impossible, comme par exemple, toujours dans Mirror's Edge, le fait de réaliser de gigantesques sauts à une centaine de mètres du sol. Il y a une grande élégance attachée à cette économie de mouvement, à la fois pratique et esthétique ; le toucher comme frappe contient en lui plus de possibilités que le geste stylisé, qui n'est qu'une mimique pour une caméra, facilement ridicule ou stéréotypique, et qui devient rapidement fatigant.

Remarquons encore que Kirkpatrick mentionnait "l'imagination» comme qualité principale du joueur pour répondre aux sollicitations de la machine. Cette mention nous intéresse tout particulièrement en ce que, lorsqu'il examinait le cheminement de l'humanité, André Leroi-Gourhan voyait dans l'avènement de l'audiovisuel un dangereux substitut à l'imagination qu'il caractérisait comme "propriété fondamentale de l'intelligence $»^{16}$. Le paléontologue français envisageait en effet une ligne d'évolution partant de l'écriture pictographique, qui laissait la pensée vagabonder, à l'écriture 
alphabétique, plus rigide mais qui laissait une marge d'interprétation personnelle, à la culture audiovisuelle dans laquelle :

[...] la marge d'expression individuelle se trouve excessivement réduite puisque le symbole et son contenu se confondent dans un réalisme qui tend vers la perfection et puisque d'autre part la situation réelle ainsi recréée laisse le spectateur hors de toute possibilité d'intervention active. ${ }^{17}$

Le rétablissement du rôle de la main non pas tant dans l'image que sur l'image est-il une brèche dans ce constat pessimiste ? Peut-être. Nous constatons en effet que Kirkpatrick envisage l'imagination au sens plus restreint de capacité à résoudre un problème de manière créative. Il faudrait alors savoir jusqu'à quel point cette définition peut être étendue, si l'imagination et la main du joueur, peuvent, de concert, se muer en une interprétation, si de l'acte physique même du jeu peut émerger un sens qui puisse différer en fonction de celui qui joue?

Répondre à ces questions par l'affirmative, c'est tout l'enjeu des mécanismes de jeu plus profonds qu'une simple action physique ou mécanique et dont on étudiera deux exemples ci-dessous. Mais quelle est la véritable part du toucher dans cette opération ? Peut-il espérer être autre chose qu'un acte préparatoire?

De plus, toutes ces pressions sur les boutons, si elles tombent, comme le geste d'un pianiste, sous le concept de corps au travail, ont une force créatrice sans doute moins profonde. Un pianiste peut avoir un toucher particulier, un art de poser les notes d'une manière qui lui soit propre. Ce toucher est certainement aidé par une dextérité sans faille, mais ne se confond pas avec cette virtuosité purement technique. En revanche le toucher vidéoludique ne semble s'élaborer que comme dextérité et non comme signature, comme marque d'une interprétation. Est-il alors possible de le développer dans cette direction?

\section{Poétique du toucher : l'émotion et le rythme}

Le caractère le plus propre de l'haptique, par rapport à l'optique, était, nous l'avons vu, une indifférenciation, une coexistence, par opposition à une hiérarchisation et à une différenciation. Or la main virtuelle est un outil de mesure vidéoludique quand la main réelle, parce qu'elle est le soubassement de l'expérience esthétique s'exclut en partie de celle-ci. La main est poïétique, mais peut-elle être à son tour et en même temps l'objet d'une poétique ? Nous aimerions évoquer ainsi deux directions possibles, une pour chaque main, qui ferait du toucher un élément central de l'expérience esthétique des jeux vidéo.

En premier lieu, Ico ${ }^{18}$ nous semble être un jeu qui met la main de l'avatar comme mécanisme haptique au centre de son fonctionnement. Dans cette œuvre, le joueur contrôle un jeune garçon né avec des cornes sur le front. Cela étant considéré comme annonciateur de malheur, il est enfermé, au début du jeu, dans un château labyrinthique pour y mourir sans attirer le mauvais œil. Il y rencontre Yorda, jeune fille fantomatique, mystérieusement emprisonnée dans une cage, qu'il libère.

Une des principales actions du jeu consiste à prendre la main de Yorda afin de la guider, de l'aider à sauter par dessus un précipice, ou de l'arracher aux griffes des mystérieuses ombres qui veulent la remettre en captivité. Pourquoi alors qualifier cette action d'haptique quand la main de l'avatar ne réagit qu'à la pression d'un bouton et que, contrairement à L.A. Noire, elle n'est jamais véritablement autonome par rapport au reste 
du corps fonctionnel ? La réponse est double et se situe tant sur le plan symbolique que plus purement vidéoludique.

Prendre la main est le geste amoureux ou filial par excellence, chargé d'affection tactile. C'est cette charge émotive simple, que n'a pas la manipulation d'un indice, qui rend le joueur sensible à la poésie de ce geste. Certes, Yorda n'est pas autre chose qu'un élément jouable dans l'espace strié du code, mais le lissage par l'image fait effet et effectue la conversion de l'optique à l'haptique que n'arrivaient pas à réaliser les mains de Faith : c'est qu'il y a ici un sens, un noyau d'interprétations qui peut prendre le pas sur le code. Mais même au niveau de sa valeur pour le système de jeu, cette action est différente de celles que nous avons envisagées jusqu'ici. La main était présente pour donner une solution : qu'elle soit sommaire comme abattre un ennemi ou attraper un recoin, ou plus subtile, ainsi la manipulation de L.A. Noire. Mais ici, prendre Yorda par la main est un acte problématique puisque cela rend le personnage plus lent et vulnérable aux attaques des fantômes. Cette impuissance partielle de l'acte virtuel est frappante car elle rappelle bien l'idée de fragilité du lien d'une main à l'autre. Là où la main qui s'accroche à un mur ou tient un pistolet est sans faille, sa faillibilité toute humaine est ici évoquée par la place même de la mécanique dans le système des règles.

31 Notre deuxième exemple est Rez, le shoot'em up musical de Sega ${ }^{19}$. Un shoot'em up est un genre de jeu qui a connu son âge d'or dans les années quatre-ving et quatre-vingt-dix, dans lequel le joueur, souvent aux commandes d'une forme de vaisseau, doit tirer sur tout ce qui bouge. Rez nous permet quant à lui, de contrôler une sorte de programme informatique anthropomorphe flottant à l'intérieur d'un réseau attaqué par des virus. Mais dans Rez, nul besoin de tuer tous les ennemis dont la plupart ne font que passer sans présenter le moindre danger.

Chaque niveau est rythmé par piste musicale qu'il s'agit de compléter à son gré en tirant sur les ennemis, chacun d'eux produisant un son particulier lorsqu'il est touché. La bande son est donc en partie générée par le joueur qui peut alors avoir à faire des choix d'interprétations : réaliser un enchaînement de sons rapides en éliminant tous les ennemis à l'écran en une salve ou bien choisir de leur tirer dessus un à un, ce qui en plus de ralentir le rythme, ne produit pas exactement les mêmes effets sonores. Peut-on alors dire qu'il y a un toucher du bon joueur de Rez comme il y a le toucher d'un bon pianiste? Non, car la palette d'intentions et de possibilités est bien plus réduite. Il semble difficile en cinq niveaux, sur cinq musiques imposées de dégager un style de jeu reconnaissable d'autant qu'il serait sûrement inapplicable à d'autres jeux. Néanmoins, par le libre choix, c'est bien l'idée d'une interprétation qui vient se superposer à l'imagination du joueur, interprétation d'autant plus profonde qu'elle est redoublée par d'autres types de sensations.

34 En effet, au-delà de l'aspect sonore, l'esthétique du jeu s'inspirait très largement des écrits théoriques de Kandinsky afin de proposer une expérience synesthésique - ainsi la destruction d'un ennemi n'est pas uniquement sonore, elle laisse aussi s'envoler une légère trace colorée. De plus l'ambition synesthésique passait aussi par le tactile. En effet, depuis le milieu des années quatre-vingt-dix, les manettes de consoles de salon sont en général équipées de dispositifs vibrants, utilisés surtout ponctuellement pour simuler une explosion ou la réception d'un saut. Ici cette vibration est continue et en rythme avec la musique. La main n'était plus dans un rôle purement constructeur mais récepteur. Rez n'est peut être pas le premier jeu vidéo à avoir reconnu que tout le corps participait à 
l'expérience vidéoludique, sinon la technique de la vibration n'aurait pas existé auparavant, mais c'est sans doute celui qui est allé aussi loin dans l'exploitation esthétique de cette ressource.

S'il faut à présent répondre à la question que nous posions dans notre titre - quelle poétique de la main y a-t-il dans les jeux vidéo - notre réponse sera double, car double est l'objet. Pour ce qui est de la main physique, on a vu qu'elle était davantage une instance poiétique qu'esthésique. L'exemple de Rez a montré néanmoins dans quelle mesure elle constituait un territoire de l'expérience à explorer.

Quant à la main virtuelle, nous avons constaté combien l'expérience du toucher était pour elle difficile dans un milieu dominé par l'optique et par l'utilisation du corps de l'avatar comme outil fonctionnel. Incorporer du toucher à cet ensemble est avant tout une affaire de choix et de possibilité d'une interaction signifiante. La leçon d'Ico prend alors une portée plus large, pour ce qui est de la séparation haptique/optique dans l'espace vidéoludique.

Nous avons envisagé en quoi la main virtuelle devait gagner en autonomie pour devenir un organe de sens, et non pas seulement un outil ou un maquillage. Cette autonomie, au fond, se définit peut-être moins par rapport au corps de l'avatar, que par rapport aux règles qui régissent le monde vidéoludique $\mathrm{Si}$ le caractère optique de l'acte vidéoludique est la discrimination entre le pertinent et le non-pertinent pour les règles, l'haptique est là quand l'action du jeu parvient à s'affranchir de cette limite - il y a autonomie de la main virtuelle quand son action n'est pas nécessairement un accomplissement.

\section{NOTES}

1. Mirror's Edge, Dice, Electronic Arts, 2009.

2. Half-Life 2, Valve Vivendi Universal Games, 2004.

3. André Leroi-Gourhan, Le geste et la parole II. La mémoire et les rythmes, Paris, Albin Michel, 1965, p. 40-41.

4. L.A. Noire, Team Bondi, Rockstar Games, 2011.

5. Gilles Deleuze \& Félix Guattari, Capitalisme et schizophrénie II. Mille Plateaux, Paris, Éditions de Minuit, 1981, pp. 614-619. Haptique vient du grec « haptomai », toucher.

6. Ibid., p. 598.

7. Sur cette idée, que nous partageons en grande partie, voir Mathieu Triclot, Philosophie des jeux vidéo, Paris, La Découverte, 2011, pp. 94-97.

8. Gilles Deleuze \& Félix Guattari, op. cit., p. 615.

9. Mathieu Triclot, op. cit., pp. 103-107.

10. Ibid., p. 104.

11. Ibid., pp. 108-110.

12. Graeme Kirkpatrick, Aesthetic Theory and the Videogame, Manchester, Manchester University Press, 2001, p. 119 et suiv.

13. Graeme Kirkpatrick, ibid., p. 137. Nous traduisons. 
14. Les performances de Dance Dance Revolution sont une exception notable mais elles nous semblent pourtant relever de la danse augmentée par ordinateur. Le dispositif numérique fait alors davantage office de compteur de score, que d'expérience purement vidéoludique.

15. Paul Valéry, "Discours prononcé au deuxième congrès international d'esthétique et de science de l'art », Variété III, Paris, Gallimard, 1936, repris dans Variété, III, IV et V, Paris, Gallimard, 2002, p. 532-533.

16. André Leroi-Gourhan, Le geste et la parole I. Technique et langage, Paris, Albin Michel, 1964, p. 296.

17. André Leroi-Gourhan, ibid., p. 295.

18. Ico, Sony, Sony, 2002.

19. Rez, UGA, Sega, 2001.

\section{RÉSUMÉS}

Le présent article s'intéresse au rôle de la main virtuelle de l'avatar autant qu'à la main physique du joueur. On y verra que la première citée n'est le plus souvent qu'un habillage cosmétique, impropre à véhiculer une sensation de toucher. La main du personnage fictionnel est happé par l'espace optique des jeux vidéo. Du côté du joueur physique, on verra comment le fait même de jouer à un jeu vidéo renvoie historiquement à la possibilité d'un toucher, d'une intimité avec l'ordinateur. Nous envisagerons les gestes de la main du joueur comme relevant du concept de corps au travail. Enfin, au travers de deux exemples de jeux vidéo, nous verrons par quels moyens le toucher peut, malgré toutes les difficultés rencontrées, être l'objet d'une expérience esthétique.

A Poetics of Hand in Video Games?

The object of this article is both the avatar's virtual hand and the player's own hand. The former is, most of the time, nothing but a cosmetic layer that is unable to convey any kind of aesthetic touch, overwhelmed by the optic nature of video game spaces. As for the physical player, we'll see how to play video games was only historically possible thanks to the creation of a personal link between the computer and the hacker. Keeping this in mind, we'll describe the hand's gesture with the concept of body at work. Finally, building upon two examples, we'll see how an aesthetic touch, despite all the previous difficulties, may be experienced.

\section{AUTEUR}

\section{THOMAS MORISSET}

Etudiant en philosophie à l'Université Paris-IV Sorbonne. Ses recherches portent sur l'esthétique des jeux vidéo. 